\title{
Temperature effects in pulsating superfluid neutron stars
}

\author{
Elena M. Kantor ${ }^{1,2}$ and Mikhail E. Gusakov ${ }^{1}$ \\ ${ }^{1}$ Ioffe Physical Technical Institute, Polytekhnicheskaya 26, 194021 St.-Petersburg, Russia \\ ${ }^{2}$ St.-Petersburg State Polytechnical University, Polytekhnicheskaya 29, 195251 St.-Petersburg, Russia
}

(Dated:)

\begin{abstract}
We study the effects of finite stellar temperatures on the oscillations of superfluid neutron stars. The importance of these effects is illustrated with a simple example of a radially pulsating general relativistic star. Two main effects are taken into account: (i) temperature dependence of the entrainment matrix and (ii) the variation of the size of superfluid region with temperature. Four models are considered, which include either one or both of these two effects. Pulsation spectra are calculated for these models, and asymptotes for eigenfrequencies at temperatures close to critical temperature of neutron superfluidity, are derived. It is demonstrated that models that allow for the temperature effect (ii) but disregard the effect (i), yield unrealistic results. Eigenfunctions for the normal- and superfluid-type pulsations are analyzed. It is shown that superfluid pulsation modes practically do not appear at the neutron-star surface and, therefore, can hardly be observed by measuring the modulation of the electromagnetic radiation from the star. The e-folding times for damping of pulsations due to the shear viscosity and nonequilibrium modified Urca processes are calculated and their asymptotes at temperatures close to the neutron critical temperature, are obtained. It is demonstrated that superfluid pulsation modes are damped by 1-3 orders of magnitude faster than normal modes.

PACS numbers: 97.60.Jd, 47.75.+f, 97.10.Sj, 47.37.+q
\end{abstract}

\section{INTRODUCTION}

High-frequency oscillations of the electromagnetic radiation, interpreted as resulting from neutron-star oscillations, have already been observed during giant flares [1, 2]. Also, detectors are being designed, which, according to preliminary estimates, will be able to detect gravitational radiation from oscillating neutron stars (NSs) $3-6]$. To interpret the existing and future observations, it is necessary to have a well-developed theory of neutron-star oscillations. Our study is devoted to the superfluid sector of this theory. To be more concrete, the goal of the present paper is to demonstrate, using radial pulsations as an example, that the effects of finite temperatures strongly affect dynamics of NSs.

Neutron stars are relativistic objects having supranuclear densities and strongly curving spacetime around them. Therefore, accurate calculations of their properties should be made in the frame of the general relativity theory. The situation is additionally complicated by the fact that baryons in the neutron-star cores can be in superfluid state 7-9]. Thus, several velocity fields can co-exist in matter without dissipation, which makes hydrodynamic equations substantially more complicated [10-17]. Epstein 18] was the first who considered sound waves in the superfluid matter of NSs. Global pulsations of superfluid Newtonian stars were first studied by Lindblom and Mendell [14]. Since then, a growing number of papers devoted to oscillations of superfluid NSs have been published (see, e.g., [10 13, 19 27] and references therein). To make the problem tractable, most of these authors employed the nonrelativistic hydrodynamics in their calculations. Pulsations of general relativistic superfluid NSs were analyzed only in Refs. [12, 13, 20 23] under a number of simplified assumptions. In particular, until recently it was customary to use the zero-temperature limit of superfluid hydrodynamics. However, as it was first shown in Ref. [12] (hereafter GA06), pulsation spectra of superfluid NSs can be very sensitive to variation of temperature.

The authors of GA06 studied oscillations of superfluid NSs using the finite-temperature version of relativistic hydrodynamics for superfluid mixtures, described in detail in Refs. 11, 12, 30, 31]. For simplicity, only radial pulsations were analyzed and the simplest npe-matter composition of the stellar core was adopted, including neutrons $(n)$, protons $(p)$, and electrons $(e)$. Neutrons and protons in the core were allowed to be superfluid, while free neutrons in the crust were treated as normal (nonsuperfluid). Owing to the fact that the relativistic entrainment matrix (analog of the superfluid density [15] for mixtures) is a steep function of temperature [32], the spectrum obtained in GA06 was strongly temperature-dependent. The authors of GA06 neglected another temperature effect, the decrease in the size of the superfluid region with increasing temperature. It should be noted that this effect has been considered in several papers [23, 28, 29]; however, the authors of these papers disregarded the temperature dependence of the entrainment matrix (that is, they used the zero-temperature limit of superfluid hydrodynamics).

In the present study we, for the first time, analyze the influence of both of these temperature effects on the pulsation spectra. For that we consider the more realistic profiles of the neutron critical temperature $T_{c n}(r)$ than that employed in GA06, where it was assumed that the red-shifted $T_{c n}$ is constant throughout the core. We also compare the 
eigenfunctions for normal and superfluid pulsation modes, and calculate their characteristic damping times. The asymptotes for the eigenfrequencies and for e-folding times at temperatures close to $T_{c n}$ will also be examined.

The paper is organized as follows. In Sec. II we briefly discuss the superfluid hydrodynamics for mixtures and write down a system of equations describing radial oscillations of general relativistic superfluid NSs. In Sec. III, we present the pulsation spectra calculated for four different neutron-star models that allow for one or both of the temperature effects (the temperature dependence of the entrainment matrix and/or the variation of the size of the superfluid region with temperature). In Sec. IV, the approximate approach of Refs. 33, 34] is used to obtain the asymptotes for pulsation eigenfrequencies at temperatures close to $T_{c n}$. Sec. V is devoted to analysis of the eigenfunctions of radial pulsations. Finally, in Sec. VI we study damping of radial pulsations due to the shear viscosity and nonequilibrium modified Urca processes. Section VII presents the summary.

In the following, unless otherwise stated, the system of units used is one in which the Boltzmann constant $k_{B}$, the Planck constant $\hbar$, and the speed of light $c$ equal unity: $k_{B}=\hbar=c=1$.

\section{BASIC EQUATIONS}

\section{A. Superfluid hydrodynamics}

Following GA06, we consider the simplest npe-matter composition of the neutron-star cores. Because both protons and neutrons can be in the superfluid state, one has to use the relativistic hydrodynamics of superfluid mixtures to study the oscillations of NSs (see, e.g., [11, 12, 30, 31]). The main distinctive feature of this hydrodynamics is the presence of several velocity fields in the mixture. In our case, these are the four-velocity $u^{\mu}$ of the 'normal' component of matter (electrons and Bogoliubov excitations of neutrons and protons) as well as the four-velocities of superfluid neutrons $v_{s(n)}^{\mu}$ and superfluid protons $v_{s(p)}^{\mu}$. Below we briefly discuss the basic equations describing nondissipative motion of superfluid mixtures (for more detail, see [11, 12, 30, 31]).

The continuity equation and the energy-momentum conservation law have the form

$$
\begin{aligned}
j_{(l) ; \mu}^{\mu} & =0, \quad j_{(i)}^{\mu}=n_{i} u^{\mu}+Y_{i k} w_{(k)}^{\mu}, \quad j_{(e)}^{\mu}=n_{e} u^{\mu}, \\
T_{; \mu}^{\mu \nu} & =0, \quad T^{\mu \nu}=(P+\varepsilon) u^{\mu} u^{\nu}+P g^{\mu \nu}+Y_{i k}\left(w_{(i)}^{\mu} w_{(k)}^{\nu}+\mu_{i} w_{(k)}^{\mu} u^{\nu}+\mu_{k} w_{(i)}^{\nu} u^{\mu}\right) .
\end{aligned}
$$

Here and below the subscripts $i$ and $k$ refer to nucleons: $i, k=n, p$; the subscript $l$ runs over all particle species, $l=n, p, e$. Unless otherwise stated the summation is assumed over the repeated nucleon indices $i, k$ and over the spacetime indices $\mu, \nu$, and $\alpha$. In Eqs. (11) and (2) $j_{(l)}^{\mu}$ is the particle four-current for species $l$; $T^{\mu \nu}$ is the energymomentum tensor; $g^{\mu \nu}$ is the metric tensor; $n_{l}$ and $\mu_{l}$ are the number density and chemical potential of particle species $l$, respectively; $\varepsilon$ and $P$ are the energy density and pressure, respectively. The four-vector $w_{(i)}^{\mu}$ is related to the superfluid four-velocity $v_{s(i)}^{\mu}$ by equality: $w_{(i)}^{\mu}=\mu_{i}\left(v_{s(i)}^{\mu}-u^{\mu}\right)$. In what follows, all the thermodynamic quantities (e.g., $n_{l}$ and $\varepsilon$ ) are defined (measured) in the reference frame comoving with the normal component of the fluid [in this frame $u^{\mu}=(1,0,0,0)$; notice that $u^{\mu}$ is normalized in such a way that $\left.u_{\mu} u^{\mu}=-1\right]$. This imposes an additional constraint on the four-vector $w_{(i)}^{\mu}[11,12]$,

$$
u_{\mu} w_{(i)}^{\mu}=0 .
$$

Using this constraint and Eqs. (11) and (2) one immediately finds that, in a relativistically invariant form, the particle number densities are defined as $n_{l}=-u_{\mu} j_{(l)}^{\mu}$ [35], while the energy density is $\varepsilon=u_{\mu} u_{\nu} T^{\mu \nu}$.

The matrix $Y_{i k}$ in Eqs. (11) and (2), named the relativistic entrainment matrix, is a generalization of the concept of superfluid density [15] to the case of relativistic mixtures. In the nonrelativistic theory, a similar matrix was first considered by Andreev and Bashkin [36]. The matrix $Y_{i k}$ is expressed in terms of the Landau parameters $F_{1}^{i k}$ of asymmetric nuclear matter and universal functions of temperature, $\Phi_{i}$, as described in [32]. Generally, it can be presented as a function of density $\rho$ and the combinations $T / T_{c n}$ and $T / T_{c p}: Y_{i k}=Y_{i k}\left(\rho, T / T_{c n}, T / T_{c p}\right)$, where $T_{c p}$ is the proton critical temperature.

For illustration, in Fig. 1 we plot the elements $Y_{i k}$ as functions of temperature $T$ for the fixed baryon number density $n_{b}=3 n_{0}=0.48 \mathrm{fm}^{-3}$. The matrix elements are normalized to the constant $Y=3 n_{0} / \mu_{n}\left(3 n_{0}\right) \approx 2.60 \times$ $10^{41} \mathrm{erg}^{-1} \mathrm{~cm}^{-3}$, where $n_{0}=0.16 \mathrm{fm}^{-3}$ is the density in atomic nuclei and $\mu_{n}\left(3 n_{0}\right)$ is the neutron chemical potential. Here and below, in all our calculations we use the parameterization [37] of equation of state (EOS) of Akmal et al. 38] (hereafter APR). The vertical dot-dashed lines indicate the critical temperatures for neutrons, $T_{c n}=6 \times 10^{8} \mathrm{~K}$, and protons, $T_{c p}=5 \times 10^{9} \mathrm{~K}$. Let us stress that the actual values of baryon critical temperatures as well as their density 


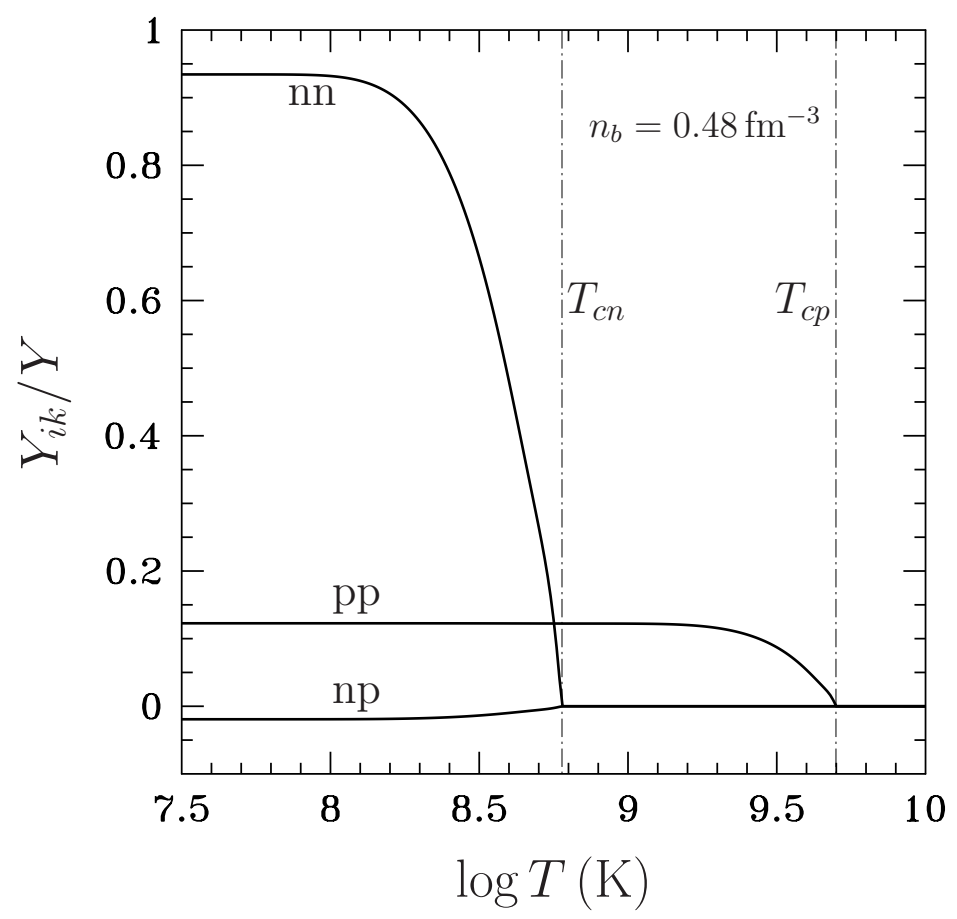

FIG. 1: Normalized symmetric matrix $Y_{i k} / Y$ as a function of $T$ for $n_{b}=3 n_{0}=0.48 \mathrm{fm}^{-3}$. The normalization constant $Y=3 n_{0} / \mu_{n}\left(3 n_{0}\right) \approx 2.60 \times 10^{41} \mathrm{erg}^{-1} \mathrm{~cm}^{-3}$. Solid lines show the elements $Y_{i k} / Y(i, k=n, p)$. Vertical dot-dashed lines indicate the baryon critical temperatures $T_{c n}=6 \times 10^{8} \mathrm{~K}$ and $T_{c p}=5 \times 10^{9} \mathrm{~K}$.

profiles are poorly known. Different authors obtain different results for $T_{c n}$ and $T_{c p}$ depending on what microphysical input they use and how they take into account the many body effects [7, 8]. Because of a large uncertainty in the models of baryon critical temperatures, in this paper we will treat them as free parameters. Our models of $T_{c n}$ and $T_{c p}$ do not contradict these, available in the literature.

One sees from Fig. 1 that at low temperatures $\left(T \lesssim 10^{8} \mathrm{~K}\right)$, the elements of the matrix $Y_{i k}$ are nearly independent of temperature and coincide with their values at $T=0$. With increasing temperature, they start to decrease and vanish after the corresponding critical temperature is reached. Thus, the matrix $Y_{i k}$ strongly depends on $T$ near the superfluid phase transition.

Along with the continuity equations and the energy-momentum conservation, one should impose an additional constraint on the superfluid velocities, following from the potentiality of superfluid motion [11, 12],

$$
\partial^{\nu}\left[w_{(i)}^{\mu}+q_{i} A^{\mu}+\mu_{i} u^{\mu}\right]=\partial^{\mu}\left[w_{(i)}^{\nu}+q_{i} A^{\nu}+\mu_{i} u^{\nu}\right]
$$

where $q_{i}$ is the charge of a species $i$, and $A^{\mu}$ is the four-potential of the electromagnetic field.

To close the system, the hydrodynamic equations should be supplemented with the second law of thermodynamics, which has the form [11, 12]

$$
d \varepsilon=T d S+\mu_{e} d n_{e}+\mu_{i} d n_{i}+\frac{Y_{i k}}{2} d\left(w_{(i)}^{\alpha} w_{(k) \alpha}\right),
$$

where $S$ is the entropy density.

\section{B. Radial pulsations}

The metric of a radially pulsating nonrotating symmetric NS has the form (see, e.g., [39]):

$$
\mathrm{d} s^{2}=-\mathrm{e}^{\nu} \mathrm{d} t^{2}+r^{2} \mathrm{~d} \Omega^{2}+\mathrm{e}^{\lambda} \mathrm{d} r^{2},
$$

where $r$ and $t$ are the radial and time coordinates, respectively; $\mathrm{d} \Omega$ is the element of a solid angle in a spherical coordinate system with the origin at the stellar center. The metric coefficients $\nu(r, t)$ and $\lambda(r, t)$ depend only on $r$ 
and $t$. In what follows, the quantities related to a star in the hydrostatic equilibrium will be denoted by the subscript ' 0 '. For example, the metric coefficients of an unperturbed star are designated as $\nu_{0}(r)$ and $\lambda_{0}(r)$.

Using Eqs. (1)-(5) the authors of GA06 derived the system of linear equations that describes radial pulsations of a general relativistic superfluid NS (all perturbations were assumed to be small and dependent on time $t$ as $\exp (i \omega t)$, where $\omega$ is the pulsation frequency):

$$
\begin{array}{r}
\mathrm{e}^{\lambda_{0}-\nu_{0}} \omega^{2} n_{b 0} \mu_{n 0} \xi_{b}=\frac{\partial \delta P}{\partial r}+\delta P \frac{\mathrm{d}}{\mathrm{d} r}\left(\frac{1}{2} \lambda_{0}+\nu_{0}\right)+ \\
\frac{1}{2} \delta \varepsilon \frac{\mathrm{d} \nu_{0}}{\mathrm{~d} r}-\frac{1}{2} n_{b 0} \mu_{n 0} \xi_{b}\left(\frac{\mathrm{d} \nu_{0}}{\mathrm{~d} r}+\frac{1}{r}\right) \frac{\mathrm{d}}{\mathrm{d} r}\left(\lambda_{0}+\nu_{0}\right) \\
\mu_{n 0} \mathrm{e}^{\lambda_{0}-\nu_{0} / 2} \omega^{2}\left(z_{n}+\xi\right)=\frac{\partial}{\partial r}\left(\delta \mu_{n} \mathrm{e}^{\nu_{0} / 2}+\frac{1}{2} \mu_{n 0} \mathrm{e}^{\nu_{0} / 2} \delta \nu\right)
\end{array}
$$

Here, the quantities $\delta \varepsilon, \delta P, \delta \mu_{n}$, and $\delta \nu$ are, respectively, perturbations of $\varepsilon, P, \mu_{n}$ and the metric coefficient $\nu(r, t)$; $\xi$ is the Lagrangian displacement of the normal liquid component, related to its velocity by [39]

$$
u^{1}=\mathrm{e}^{-\nu_{0} / 2} \frac{\partial \xi}{\partial t}=i \omega \mathrm{e}^{-\nu_{0} / 2} \xi
$$

Furthermore, $z_{i}$ is the Lagrangian displacement of a superfluid component $i$ relative to the normal component, defined as

$$
w_{(i)}^{1}=\mu_{i 0} \mathrm{e}^{-\nu_{0} / 2} \frac{\partial z_{i}}{\partial t}=i \omega \mu_{i 0} \mathrm{e}^{-\nu_{0} / 2} z_{i}
$$

and the function $\xi_{b}$ is proportional to the baryon current density $j_{(b)}^{\mu}=n_{b} u^{\mu}+Y_{n k} w_{(k)}^{\mu}+Y_{p k} w_{(k)}^{\mu}$ [see Eq. (1)], and is given by

$$
\xi_{b}=\xi+\frac{\mu_{i 0} Y_{n i} z_{i}}{n_{b 0}}+\frac{\mu_{i 0} Y_{p i} z_{i}}{n_{b 0}}
$$

Equation (7) is the relativistic Euler equation generalized to the case of superfluid stars. A similar equation has been derived for normal matter by Chandrasekhar [39]. Equation (8), specific to superfluid matter, is a consequence of the potentiality of superfluid motion [see condition (4)].

Because we are interested in oscillations whose frequencies are substantially smaller than the electron plasma frequency, one can assume that the quasineutrality condition $n_{p}=n_{e}$ is always satisfied. As a consequence, the electron and proton currents must be equal, $j_{(p)}^{\mu}=j_{(e)}^{\mu}$. Using now expression (11) and the definition (10), one obtains an additional constraint that relates $z_{n}$ and $z_{p}$,

$$
\mu_{k 0} Y_{p k} z_{k}=0
$$

In this study, it will be convenient to transform Eq. (8) and rewrite it in a somewhat different form. For this purpose, we multiply Eq. (8) by $n_{b 0} \exp \left(-\nu_{0} / 2\right)$ and subtract Eq. (7) from the result. After some algebra one gets, using equation (54) for $\partial \delta \nu / \partial r$ from GA06 (see also [33, 34]),

$$
\mu_{n 0} n_{b 0} \mathrm{e}^{\lambda_{0}-\nu_{0}} \omega^{2}\left(z_{n}-\frac{\mu_{k 0} Y_{n k} z_{k}}{n_{b 0}}\right)=n_{e 0} \frac{\partial \delta \mu}{\partial r},
$$

where $\delta \mu \equiv \mu_{n}-\mu_{p}-\mu_{e}$ is the disbalance of chemical potentials. Solving the system of equations (7), (12), and (13), we can find the eigenfunctions $\xi, z_{n}$, and $z_{p}$, as well as the spectrum of eigenfrequencies $\omega$. In order to do so, it is, however, necessary to express the quantities $\delta \varepsilon, \delta P$, and $\delta \mu$ in terms of the functions $\xi$, $z_{n}$, and $z_{p}$ and to formulate the boundary conditions for Eqs. (7) and (13).

Owing to the quasineutrality condition, $n_{e}=n_{p}$, any thermodynamic quantity can be presented as a function of two variables, say, $n_{b}$ and $n_{e}$ (the dependence on the quadratically small scalars $w_{(i)}^{\mu} w_{(k) \mu}$ and on $T$ can be neglected). Because we consider small-amplitude pulsations, the pressure perturbation and the chemical potential disbalance can be expanded in Taylor series near their equilibrium values,

$$
\begin{aligned}
\delta P & =\frac{\partial P\left(n_{b 0}, n_{e 0}\right)}{\partial n_{b 0}} \delta n_{b}+\frac{\partial P\left(n_{b 0}, n_{e 0}\right)}{\partial n_{e 0}} \delta n_{e} \\
\delta \mu & =\frac{\partial \delta \mu\left(n_{b 0}, n_{e 0}\right)}{\partial n_{b 0}} \delta n_{b}+\frac{\partial \delta \mu\left(n_{b 0}, n_{e 0}\right)}{\partial n_{e 0}} \delta n_{e}
\end{aligned}
$$


Similarly, in view of the beta-equilibrium condition $\delta \mu_{0}=\mu_{n 0}-\mu_{p 0}-\mu_{e 0}=0$, valid for an unperturbed star, one has from Eq. (5) (see also GA06),

$$
\delta \varepsilon=\mu_{n 0} \delta n_{b}
$$

Using continuity equations (11), the variations $\delta n_{b}$ and $\delta n_{e}$ appearing in Eqs. (14)-(16) can be expressed in terms of the Lagrangian displacements $\xi, z_{n}$, and $z_{p}$,

$$
\begin{aligned}
\delta n_{b} & =-\frac{\mathrm{e}^{\nu_{0} / 2}}{r^{2}} \frac{\partial}{\partial r}\left(r^{2} n_{b 0} \xi_{b} \mathrm{e}^{-\nu_{0} / 2}\right), \\
\delta n_{e} & =-\frac{\mathrm{e}^{\nu_{0} / 2}}{r^{2}} \frac{\partial}{\partial r}\left(r^{2} n_{e 0} \xi \mathrm{e}^{-\nu_{0} / 2}\right) .
\end{aligned}
$$

\section{Boundary conditions}

Prior to formulating the boundary conditions for Eqs. (77) and (13), we should refine the model of a star whose pulsations we analyze. We consider a two-layer model constituted by two regions: an inner region, in which neutrons are superfluid, and an outer region, in which neutrons are normal. In the inner region (for which $r \leq R_{0}$ ), the functions $z_{i}$ are nonzero; we name this region 'superfluid'. In the outer region (for which $r>R_{0}$ ), all matter components move with the same velocity $u^{\mu}$, i.e., the fluid demonstrates a nonsuperfluid behavior [even if protons remain superfluid, it follows from (12) that $\left.z_{p}=0\right]$; we name such a region 'normal'. In addition, we assume that the thermodynamic functions (e.g., the particle number densities $n_{l}$ ) do not experience any abrupt change at the superfluid-normal interface. Then one can formulate the following boundary conditions for such a stellar model (see also GA06).

(1) Vanishing of the pressure at the stellar surface.

$$
\left[\delta P+\frac{\mathrm{d} P_{0}}{\mathrm{~d} r} \xi_{b}\right]_{r=R}=0
$$

where $R$ is the circumferential radius of the star.

(2) Existence of all the derivatives with respect to $r$ at the stellar center, which means that the following limits must be finite

$$
\lim _{r \rightarrow 0} \xi_{b} / r<\infty, \quad \lim _{r \rightarrow 0} \mu_{i 0} Y_{n i} z_{i} / r<\infty .
$$

(3) Continuity of the energy current density across the superfluid-normal interface. As shown in GA06, this current is directed along the radial coordinate and is proportional to $n_{b 0} \mu_{n 0} \xi+\mu_{n 0} \mu_{i 0} Y_{n i} z_{i}=n_{b 0} \mu_{n 0} \xi_{b}$. Hence, because of the continuity of the thermodynamic functions, one has

$$
\left[\xi_{b}\right]_{r=R_{0}-0}=\left[\xi_{b}\right]_{r=R_{0}+0} .
$$

(4) Continuity of the electron current density across the superfluid-normal interface, $\xi\left(R_{0}-0\right)=\xi\left(R_{0}+0\right)$, which, combined with Eqs. (11), (12), and (21), leads to

$$
\left.\mu_{i 0} Y_{n i} z_{i}\right|_{r=R_{0}}=0 .
$$

The solution to the system of equations (7), (12), and (13) must satisfy these four boundary conditions.

\section{PULSATION SPECTRA}

Using Eqs. (7), (12), and (13), we determined the spectrum of eigenfrequencies $\omega$ of a radially pulsating NS as a function of its internal temperature $T$. In our numerical calculations, we considered NS with the mass $M=1.4 M_{\odot}$, circumferential radius $R=12.17 \mathrm{~km}$, and central density $\rho_{\mathrm{c}}=9.26 \times 10^{14} \mathrm{~g} \mathrm{~cm}^{-3}$. We used APR EOS in the core, and Negele and Vautherin EOS in the crust [40]. For the chosen model of a star, the core-crust interface lies at a distance $R_{\mathrm{cc}}=10.88 \mathrm{~km}$ from the stellar center. Following GA06, free neutrons in the inner crust were assumed to be normal, while in the core both nucleon species were allowed to be superfluid.

To simplify calculations, the red-shifted critical temperature of protons was chosen to be constant throughout the core (i.e., independent of the density $\rho$ ) and equal to $T_{c p}^{\infty} \equiv T_{c p} \mathrm{e}^{\nu_{0} / 2}=5 \times 10^{9} \mathrm{~K}$. Note that, the pulsation spectrum 
only weakly depends on a particular form of the function $T_{c p}^{\infty}(\rho)$ and even on whether or not protons are superfluid. The point is the only quantity in superfluid hydrodynamics, which depends on the critical temperatures $T_{c n}$ and $T_{c p}$, is the entrainment matrix $Y_{i k}$. The element $Y_{n n}$ of the matrix is mostly determined by the neutron critical temperature $T_{c n}$ and is nearly insensitive to $T_{c p}$ [32]. By contrast, the elements $Y_{p p}$ and $Y_{n p}$ strongly vary with $T_{c p}$, but the terms, depending on these matrix elements, are small in pulsation equations and can be neglected to a good approximation.

To illustrate this statement let us notice that, as follows from Eqs. (7) and (13), $Y_{n p}$ appears there only in the combination $\mu_{n} Y_{n n} z_{n}+\mu_{p} Y_{n p} z_{p}$ (and $Y_{p p}$ does not explicitly enter these equations). From the quasineutrality condition (12), we obtain $z_{p}=-\mu_{n} Y_{n p} z_{n} /\left(\mu_{p} Y_{p p}\right)$, and, therefore, the second term in this combination differs from the first one by a factor $Y_{n p}^{2} /\left(Y_{n n} Y_{p p}\right)$. As follows from Fig. 1, $Y_{n p}^{2} /\left(Y_{n n} Y_{p p}\right) \sim 0.01$ and, consequently, this term can be indeed neglected.

The red-shifted temperature of unperturbed star, $T^{\infty}=T \mathrm{e}^{\nu_{0} / 2}$, was assumed to be finite and constant throughout the stellar core. The latter is a necessary condition for a superfluid star to be in the hydrostatic equilibrium [see equation (30) of GA06]. The influence of finite internal temperature on the pulsations of NSs is twofold. First, it determines the entrainment matrix $Y_{i k}(T)$ (see Fig. 1). Second, it regulates the size $R_{0}$ of the superfluid region, for which $T \leq T_{c n}(\rho)$ : the higher the temperature, the smaller the region (see Fig. 2).

We considered four models showing different behavior of $T_{c n}(\rho)$ and allowing for one or both of these temperature effects.

Model 1. The red-shifted neutron critical temperature $T_{c n}^{\infty}$ is density independent and equals $T_{c n}^{\infty}=T_{c n} \mathrm{e}^{\nu_{0} / 2}=$ $6 \times 10^{8} \mathrm{~K}$. This model assumes that superfluid region coincides with the stellar core at $T^{\infty} \leq T_{c n}^{\infty}$. Within this region we use the finite-temperature hydrodynamics, i.e., take into account the full dependence of $Y_{i k}$ on $T$. This model is identical to the model considered in GA06. The only difference is that the authors of GA06 used an approximate expression for the matrix $Y_{i k}$ [see their equation (17)], whereas in the present study we use results of a self-consistent relativistic calculation, performed in [32]. However, one can check that the expression for $Y_{i k}$ from GA06 is a good approximation for calculation of the pulsation spectrum.

Model 2. $T_{c n}^{\infty}$ depends on density and has a maximum at the stellar center, $T_{c n}^{\infty}(r=0)=6 \times 10^{8} \mathrm{~K}$. The size of the superfluid region is temperature dependent and the boundary $R_{0}$ of this region is determined by the condition

$$
T_{c n}^{\infty}\left(R_{0}\right)=T^{\infty} .
$$

However, within the superfluid region we use the zero-temperature hydrodynamics, that is, the entrainment matrix in this model does not depend on $T, Y_{i k}=Y_{i k}(T=0)$. This model has been used in a number of studies [23, 28, 29]. In particular, in Ref. 23] it was claimed that the model adequately accounts for the main temperature effects.

Model 3. The same as model 2, but within the superfluid region we use a hydrodynamics which is valid at finite temperatures; $Y_{i k}$ is temperature dependent.

Model 4 . The same as model 3 , but with $T_{c n}^{\infty}$ having a maximum at a density exceeding the central density $\rho_{c}$. At the stellar center, $T_{c n}^{\infty}(0)=6 \times 10^{8} \mathrm{~K}$.

It should be stressed that, for each of these models, the results obtained below in this paper will remain qualitatively the same if we choose another neutron critical temperature $T_{c n}^{\infty}(0)$ in the stellar center or if we vary the slope of the density profile $T_{c n}^{\infty}(\rho)$ (for models 2, 3, and 4). The only important thing that discriminates between the models and that should not be altered is the condition $\left.\left[\mathrm{d} T_{c n}^{\infty}(\rho) / \mathrm{d} \rho\right]\right|_{r=0}=0$ for models 2 and 3 and $\left.\left[\mathrm{d} T_{c n}^{\infty}(\rho) / \mathrm{d} \rho\right]\right|_{r=0}>0$ for model 4. As it will be demonstrated in Secs. III and IV, this condition influences the pulsation spectra and the asymptotes for eigenfrequencies at $T^{\infty} \rightarrow T_{c n}^{\infty}(0)$.

For illustration, Fig. 2 shows the red-shifted $T_{c n}^{\infty}$ (dashed curve) and the local $T_{c n}$ (solid curve) neutron critical temperatures as function of density $\rho$ (left panel for models 2 and 3; right panel for model 4). Dot-dashed lines indicate the central density of the star and the density at the crust-core interface. Notice that, for all the models, a sufficiently cold star (at $T^{\infty} \leq 6 \times 10^{8} \mathrm{~K}$ ) consists of superfluid inner and normal outer regions.

In Fig. 3, for each of the four models, we present an eigenfrequency spectrum of a radially pulsating superfluid NS. The alternate solid and dashed lines show eigenfrequencies $\omega$ (in units of $\omega_{0} \equiv c / R$ ) as functions of $T^{\infty}$ for the first six pulsation modes. The dot-dashed line indicates the red-shifted neutron critical temperature at the stellar center, $T_{c n}^{\infty}(0)=6 \times 10^{8} \mathrm{~K}$. The dotted lines turning into thin solid lines at a temperature $T^{\infty}>T_{c n}^{\infty}(0)$ show first three radial oscillation modes of a normal star (I, II, and III). The spectrum was not plotted in the shaded region.

It can be seen from the figure that at $T^{\infty}<T_{c n}^{\infty}(0)$ there exist two types of oscillations. Oscillation frequencies of the first type are nearly temperature independent and well coincide with the corresponding eigenfrequencies of a nonsuperfluid star of the same mass (we name this type of modes 'normal'). Oscillation frequencies of the second type strongly vary with temperature (for $T \gtrsim 5 \times 10^{7}-10^{8} \mathrm{~K}$ ); the corresponding modes are named 'superfluid'. With growing $T$, neighboring modes become closer and closer until they form avoided crossing. Near such avoided crossings any superfluid mode turns into a normal mode and vice versa. At low enough temperatures $\left(T \lesssim 5 \times 10^{7}\right.$ $\mathrm{K}$ for models 2,3 , and 4 , and $T \lesssim 10^{8} \mathrm{~K}$ for model 1 ), the eigenfrequencies cease to depend on $T$ and reach their asymptotic values. The reason for that is, first, the matrix $Y_{i k}$ becomes temperature independent at $T^{\infty} \lesssim 10^{8} \mathrm{~K}$ 

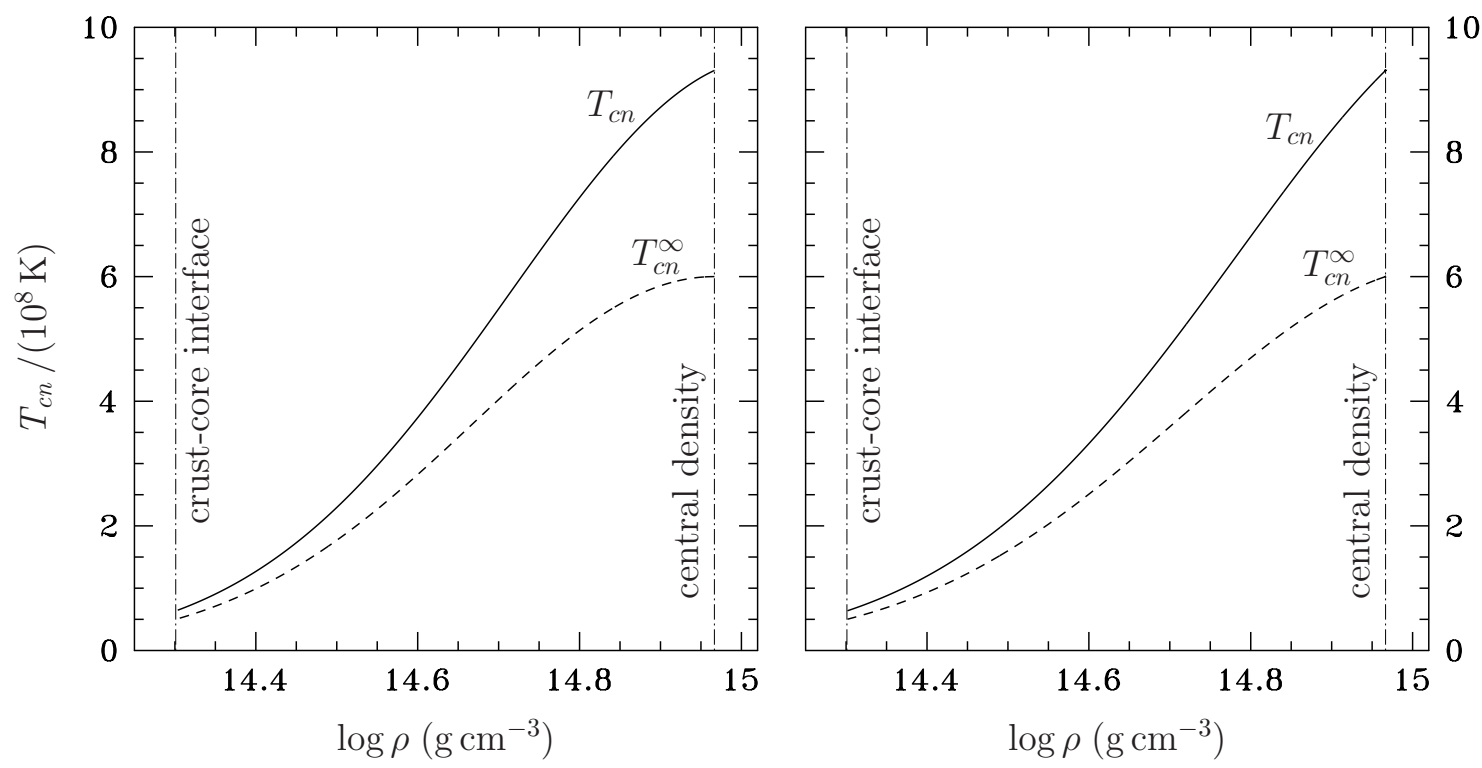

FIG. 2: Neutron critical temperatures $T_{c n}^{\infty}$ (dashed lines) and $T_{c n}$ (solid lines) versus $\rho$ (left panel for models 2 and 3 , right panel for model 4). Dot-dashed lines show the central density and crust-core interface.

and second, the radius of the superfluid region reaches its maximum value (corresponding to the core-crust interface) at $T^{\infty}=5 \times 10^{7} \mathrm{~K}$ and stops to vary as $T$ is lowered further.

For the superfluid type of pulsations, the decrease in the size $R_{0}$ of the superfluid region with increasing $T^{\infty}$ leads to a rise of the eigenfrequencies $\omega$ (this is clearly seen for model 2 and stems from the fact that, as shown below, $\left.\omega \propto 1 / R_{0}\right)$. At the same time, $Y_{n k} \rightarrow 0$ with increasing $T$ (Fig. 1), which results in decreasing of $\omega$ (this is exemplified by model 1 and follows from the fact that $\omega \propto \sqrt{Y_{n n}}$ at $T^{\infty} \rightarrow T_{c n}^{\infty}(0)$, see below). In the models 3 and 4 , these two effects compete. Asymptotes for the eigenfrequencies at $T^{\infty} \rightarrow T_{c n}^{\infty}(0)$ will be studied in detail in Sec. IVB. At temperatures higher than $T_{c n}^{\infty}(0)$, the star pulsates as a normal one [even if protons still remain superfluid, all the liquid components move together by virtue of the quasineutrality condition (12)].

As follows from Fig. 3, the pulsation spectra differ substantially for different models. For example, for most realistic models 3 and 4 in the limit $T^{\infty} \rightarrow T_{c n}^{\infty}(0)$, the eigenfrequencies either vanish (model 3), or decrease, approaching some certain finite values (model 4). The eigenfrequencies for model 1 behave similar to those for model 3. At the same time, the eigenfrequencies for model 2 behave in a drastically different way. At $T^{\infty} \rightarrow T_{c n}^{\infty}(0)$ they grow rather than decrease and tend to infinity as $R_{0} \rightarrow 0$. This example clearly demonstrates that it is insufficient just to vary the size of the superfluid region with $T$, using the zero-temperature hydrodynamics (as in model 2). Such an approach gives qualitatively incorrect spectra. Thus, it is of principal importance to take into account the temperature dependence of the entrainment matrix.

\section{EIGENFREQUENCIES AT $T^{\infty} \rightarrow T_{c n}^{\infty}(0)$}

\section{A. Approximate splitting of pulsation equations}

As shown in Refs. [33, 34], the equations that govern global pulsations of superfluid NSs can generally be represented as two weakly coupled systems of equations. The coupling parameter $s$ for these systems is given by

$$
s=\frac{n_{e 0}}{n_{b 0}} \frac{\partial P\left(n_{b 0}, n_{e 0}\right) / \partial n_{e 0}}{\partial P\left(n_{b 0}, n_{e 0}\right) / \partial n_{b 0}}
$$

and is small for realistic equations of state, $|s| \sim 0.01-0.05$. In particular, $s$ for APR EOS is shown in Fig. 4. In the case of radial pulsations, the smallness of the parameter $s$ means that the pulsation equations (77) and (13) can be treated as independent to a very good approximation (see [33, 34]). If we assume $s=0$, then Eq. (7) can be 

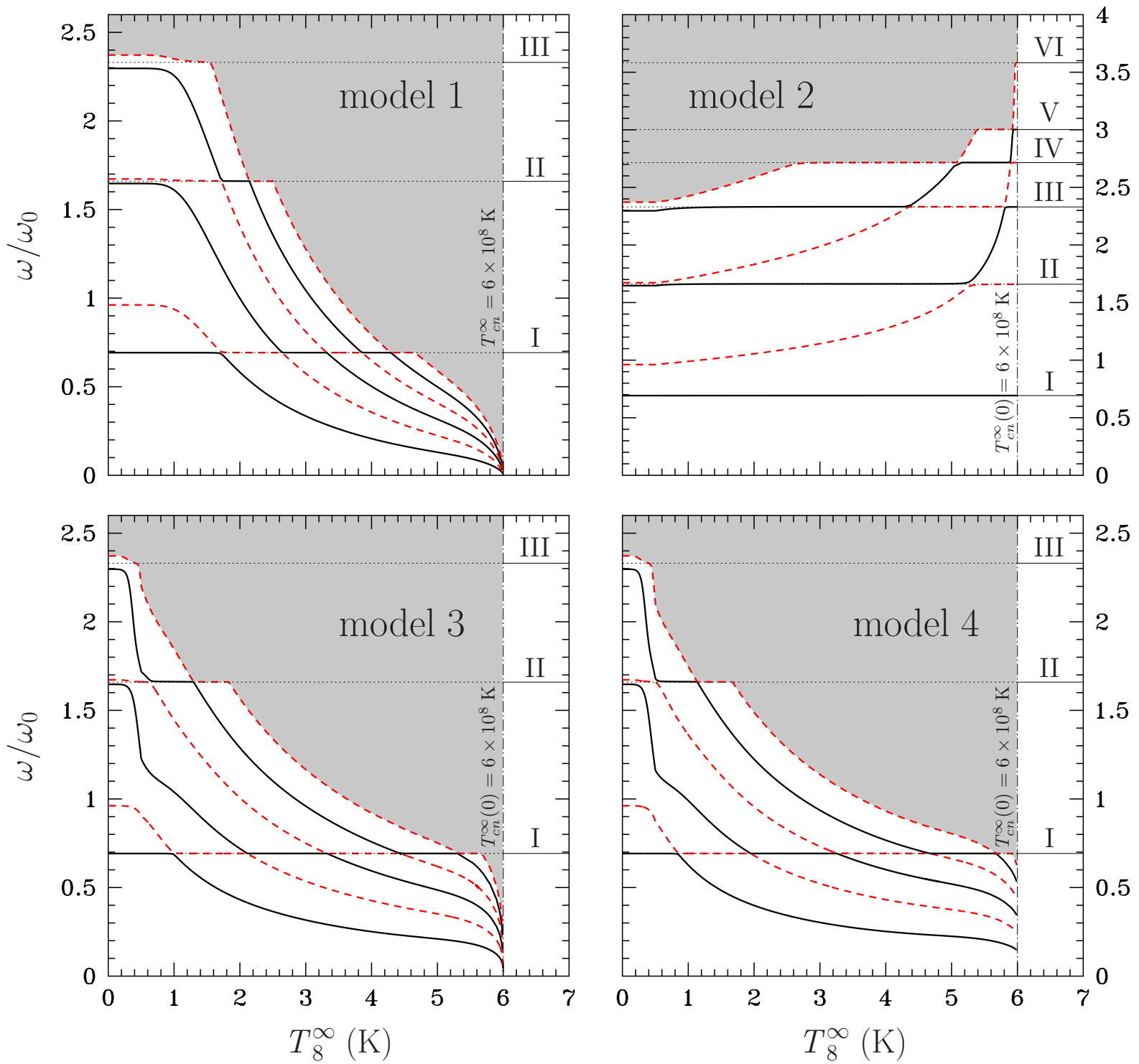

FIG. 3: Eigenfrequencies $\omega$ (in units of $\omega_{0}=c / R$ ) versus $T_{8}^{\infty}=T^{\infty} / 10^{8}$, calculated for models $1,2,3$, and 4 (see the text). Solid and dashed curves show the first six pulsation modes. The red-shifted neutron critical temperature $T_{c n}^{\infty}(0)$ in the stellar center is indicated by the vertical dot-dashed lines; the horizontal dotted lines turning into solid lines at $T^{\infty}>T_{c n}^{\infty}(0)$ show the first three eigenfrequencies (I, II, III) of a normal star of the same mass (notice that, for model 2 we show the first six eigenfrequencies of a normal star). No spectrum was plotted in the shaded region.

separated from Eq. (13) and written only in terms of the variable $\xi_{b}$ :

$$
\begin{array}{r}
\mathrm{e}^{\lambda_{0}-\nu_{0}} \omega^{2} n_{b 0} \mu_{n 0} \xi_{b}=\frac{\partial \delta P_{\text {norm }}}{\partial r}+\delta P_{\text {norm }} \frac{\mathrm{d}}{\mathrm{d} r}\left(\frac{1}{2} \lambda_{0}+\nu_{0}\right) \\
+\frac{1}{2} \delta \varepsilon \frac{\mathrm{d} \nu_{0}}{\mathrm{~d} r}-\frac{1}{2} n_{b 0} \mu_{n 0} \xi_{b}\left(\frac{\mathrm{d} \nu_{0}}{\mathrm{~d} r}+\frac{1}{r}\right) \frac{\mathrm{d}}{\mathrm{d} r}\left(\lambda_{0}+\nu_{0}\right)
\end{array}
$$

where

$$
\begin{array}{r}
\delta P_{\text {norm }} \equiv \frac{\partial P\left(n_{b 0}, n_{e 0}\right)}{\partial n_{b 0}} \delta n_{b}+\frac{\partial P\left(n_{b 0}, n_{e 0}\right)}{\partial n_{e 0}} \delta n_{e \text { norm }} \\
\delta n_{e \text { norm }} \equiv-\frac{\mathrm{e}^{\nu_{0} / 2}}{r^{2}} \frac{\partial}{\partial r}\left(r^{2} n_{e 0} \xi_{b} \mathrm{e}^{-\nu_{0} / 2}\right)
\end{array}
$$




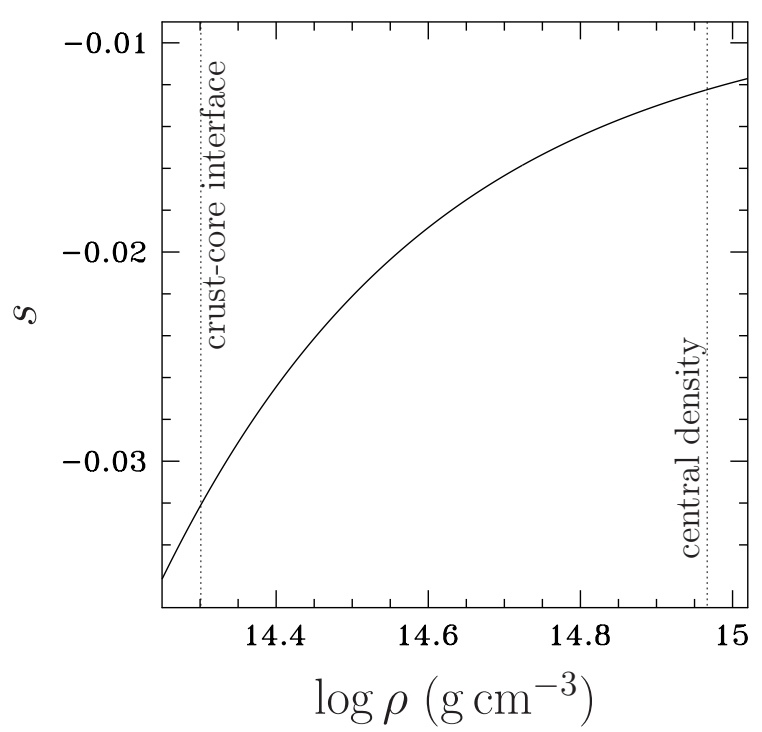

FIG. 4: Coupling parameter $s$ versus $\rho$ for APR EOS. The vertical dotted lines indicate the central stellar density and the crust-core interface.

In this form, the equation fully coincides with that describing oscillations of a nonsuperfluid star. Its solutions are eigenfrequencies $\omega$ and eigenfunctions $\xi_{b}$ of a normal star. Thus, Eq. (25) approximately describes the normal modes of oscillations in our problem. As for the superfluid modes, they are described by an equation depending only on 'superfluid' Lagrangian displacements $z_{n}$ and $z_{p}$ [33, 34],

$$
\mu_{n 0} n_{b 0} \mathrm{e}^{\lambda_{0}-\nu_{0}} \omega^{2}\left(z_{n}-\frac{\mu_{k 0} Y_{n k} z_{k}}{n_{b 0}}\right)=n_{e 0} \frac{\partial \delta \mu_{\mathrm{sf}}}{\partial r}
$$

where

$$
\delta \mu_{\mathrm{sfl}} \equiv \frac{\partial \delta \mu}{\partial n_{e}} \frac{\mathrm{e}^{\nu_{0} / 2}}{r^{2}} \frac{\partial}{\partial r}\left(r^{2} \frac{n_{e 0}}{n_{b 0}} \mu_{i 0} Y_{n i} z_{i} \mathrm{e}^{-\nu_{0} / 2}\right) .
$$

Eq. (27) can be derived from (13) if we put $\xi_{b}=0$ in the latter. Indeed, if some frequency is an eigenfrequency for the system of equations (13) and (25), but is not an eigenfrequency for Eq. (25), then this is only possible if $\xi_{b}$, the eigenfunction of Eq. (25), identically vanishes. (This consideration is strictly valid when $s=0$. For small but finite $s$ one has $\left.\xi_{b} \ll \xi \approx-\mu_{i 0} Y_{n i} z_{i} / n_{b 0}.\right)$

The approximation of independent Eqs. (25) and (27) well reproduces (with an accuracy of $1.5-2 \%$ ) the frequency spectrum of a pulsating NS. However, this approximation does not describe avoided crossings of neighboring modes (see Fig. 3). The reason is near these points Eqs. (7) and (13) are strongly interacting [33, 34], so that approximate treatment based on the decoupled Eqs. (25) and (27) is unjustified; instead of avoided crossings, Eqs. (25) and (27) predict crossings of modes. It should be noted that the existence of two types of modes, superfluid and normal, has been noted previously by a number of authors (see, e.g., Refs. [14, 28, 41]). However, an adequate analytical interpretation this fact first received in Ref. [33].

\section{B. Asymptotes for eigenfrequencies}

Using the $s=0$ approximation described above, let us consider the asymptotic behavior of eigenfrequencies at $T^{\infty} \rightarrow T_{c n}^{\infty}(0)$. For all of the four models, any given mode experiences superfluid-type pulsations near the neutron critical temperature and, therefore, to derive the asymptotes it is necessary to use Eq. (27).

As the temperature approaches the critical value $T_{c n}$, the elements $Y_{n i}\left(\rho, T / T_{c n}, T / T_{c p}\right)$ of the entrainment matrix tend to vanish according to the law [32, 42] : $Y_{n i}=F_{n i}\left(1-T / T_{c n}\right)$, where $F_{n i}$ is some (known) function of $\rho, T / T_{c n}$, and $T / T_{c p}$, which are in turn the functions of radial coordinate $r$. Taking into account that $T / T_{c i}=T^{\infty} / T_{c i}^{\infty}$, the asymptote for $Y_{n i}$ can be rewritten as

$$
Y_{n i}=F_{n i}\left(r, T^{\infty}\right)\left(1-T^{\infty} / T_{c n}^{\infty}\right) \rightarrow 0 .
$$


Owing to the smallness of the matrix elements $Y_{n i}$ near the neutron critical temperature, one can neglect the second term in the left-hand side of Eq. (27) when analyzing asymptotes for models 1, 3, and 4.

In addition, the size $R_{0}$ of the superfluid region in models 2,3 , and 4 decreases with increasing temperature and vanishes at $T^{\infty}=T_{c n}^{\infty}(0)$. The limiting behavior $R_{0}\left(T^{\infty}\right)$ can be found from the condition (23) and is determined by the profile of the neutron critical temperature. Expanding $T_{c n}^{\infty}(\rho)$ in the Taylor series in the vicinity of the stellar center and taking into account that for models 2 and $\left.3\left[\mathrm{~d} T_{c n}^{\infty} / \mathrm{d} \rho\right]\right|_{r=0}=0$ while for model $\left.4\left[\mathrm{~d} T_{c n}^{\infty} / \mathrm{d} \rho\right]\right|_{r=0} \neq 0$, one gets, for models 2 and 3 ,

$$
T_{c n}^{\infty}(r)-T_{c n}^{\infty}(0) \propto\left[\rho(r)-\rho_{c}\right]^{2} \propto r^{4}
$$

and for model 4 ,

$$
T_{c n}^{\infty}(r)-T_{c n}^{\infty}(0) \propto \rho(r)-\rho_{c} \propto r^{2} .
$$

To obtain the asymptotes (30) and (31) we used the fact that in the stellar center $\left.[\mathrm{d} \rho / \mathrm{d} r]\right|_{r=0}=0$. Now, using Eqs. (30) and (31) and the condition (23), one finds how $R_{0}$ scales with $T^{\infty}$ at $T^{\infty} \rightarrow T_{c n}^{\infty}(0)$. For models 2 and 3

$$
R_{0} \propto\left[1-T^{\infty} / T_{c n}^{\infty}(0)\right]^{1 / 4}
$$

while for model 4

$$
R_{0} \propto\left[1-T^{\infty} / T_{c n}^{\infty}(0)\right]^{1 / 2} .
$$

Making use of Eqs. (29)-(33), it is possible to analyze the dependence $\omega\left(T^{\infty}\right)$ at $T^{\infty} \rightarrow T_{c n}^{\infty}(0)$ for different models. We start with the model 1. Then, the only quantities in Eq. (27) that depend on $T$ are the matrix elements $Y_{n i}$ and the frequency $\omega$ (we remind that the size $R_{0}$ of the superfluid region is fixed for model 1). Using Eq. (29) and the fact that $T^{\infty} / T_{c n}^{\infty}$ does not depend on $r$ for this model, one can factor the temperature dependence of $Y_{n i}$ outside the differentiation sign on the right-hand side of Eq. (27). The result is

$$
\mu_{n 0} n_{b 0} \mathrm{e}^{\lambda_{0}-\nu_{0}} \omega^{2} z_{n}=n_{e 0}\left(1-\frac{T^{\infty}}{T_{c n}^{\infty}}\right) \frac{\partial}{\partial r}\left[\frac{\partial \delta \mu}{\partial n_{e}} \frac{\mathrm{e}^{\nu_{0} / 2}}{r^{2}} \frac{\partial}{\partial r}\left(r^{2} \frac{n_{e 0}}{n_{b 0}} \mu_{i 0} F_{n i}\left(r, T^{\infty}\right) z_{i} \mathrm{e}^{-\nu_{0} / 2}\right)\right] .
$$

The function $F_{n i}\left(r, T^{\infty}\right) \approx F_{n i}\left(r, T_{c n}^{\infty}\right)$ in the immediate vicinity of $T_{c n}^{\infty}$. Taking this into account, one immediately obtains from Eq. (34),

$$
\omega \propto \sqrt{1-T^{\infty} / T_{c n}^{\infty}} \rightarrow 0, \quad T^{\infty} \rightarrow T_{c n}^{\infty} .
$$

Now let us inspect model 2. This model assumes that the matrix $Y_{i k}$ is temperature independent, while $R_{0}$ decreases with increasing $T$. In that case, the asymptote for the function $\omega\left(T^{\infty}\right)$ can be determined by introducing a new dimensionless variable, $\tilde{r} \equiv r / R_{0}$, which varies within the range from 0 to 1 . Rewriting Eq. (27) employing the new variable, one finds [notice that we retain the second term on the left-hand side of Eq. (27) for this model]

$$
\mu_{n 0} n_{b 0} \mathrm{e}^{\lambda_{0}-\nu_{0}} \omega^{2} R_{0}^{2}\left(z_{n}-\frac{\mu_{k 0} Y_{n k} z_{k}}{n_{b 0}}\right)=n_{e 0} \frac{\partial}{\partial \tilde{r}}\left[\frac{\partial \delta \mu}{\partial n_{e}} \frac{\mathrm{e}^{\nu_{0} / 2}}{\tilde{r}^{2}} \frac{\partial}{\partial \tilde{r}}\left(\tilde{r}^{2} \frac{n_{e 0}}{n_{b 0}} \mu_{i 0} Y_{n i} z_{i} \mathrm{e}^{-\nu_{0} / 2}\right)\right] .
$$

At $T^{\infty}$ sufficiently close to $T_{c n}^{\infty}(0)$, when the size $R_{0}$ of the superfluid region is rather small, such functions as $\mu_{i 0}$, $Y_{i k}, n_{i 0}$, and $\nu_{0}$ are almost independent of the radius $r$ [so that one can put, e.g., $\mu_{i 0}(r) \approx \mu_{i 0}(0)$ ]. Thus, the only quantities varying with temperature in Eq. (36) are $\omega$ and $R_{0}$, and, therefore, the asymptote takes the form

$$
\omega \propto \frac{1}{R_{0}} \propto \frac{1}{\left[1-T^{\infty} / T_{c n}^{\infty}(0)\right]^{1 / 4}} \rightarrow \infty, \quad T^{\infty} \rightarrow T_{c n}^{\infty}(0) .
$$

To obtain this asymptote we also used Eq. (32).

Finally, let us consider models 3 and 4 . In these models, both $Y_{i k}$ and $R_{0}$ depend on $T^{\infty}$. So, we need to combine our reasoning for the first and second models. The pulsation equation for models 3 and 4 is given by the same Eq. (36) but without the second term $\propto Y_{n i}$ on its left-hand side; as it is argued above in this section, this term is small at $T^{\infty} \rightarrow T_{c n}^{\infty}$ and can be omitted. Again, as for model 2, in Eq. (36) one can replace such quantities as $\mu_{i 0}(r)$ by their values $\mu_{i 0}(0)$ in the stellar center. The same, of course, cannot be done for the matrix element $Y_{n i}\left(r, T^{\infty}\right)=F_{n i}\left(r, T^{\infty}\right)\left[1-T^{\infty} / T_{c n}^{\infty}(r)\right]$, because it changes substantially in the superfluid region. Using Eqs. (23) and (29) -(31) it is easy to verify that $Y_{n i}\left(r, T^{\infty}\right)$ can generally be presented as

$$
Y_{n i}=F_{n i}\left(0, T_{c n}^{\infty}(0)\right)\left[1-T^{\infty} / T_{c n}^{\infty}(0)\right]\left(1-\tilde{r}^{4}\right)+O\left(\left[1-T^{\infty} / T_{c n}^{\infty}(0)\right]^{2}\right)
$$


for model 3 and as

$$
Y_{n i}=F_{n i}\left(0, T_{c n}^{\infty}(0)\right)\left[1-T^{\infty} / T_{c n}^{\infty}(0)\right]\left(1-\tilde{r}^{2}\right)+O\left(\left[1-T^{\infty} / T_{c n}^{\infty}(0)\right]^{2}\right)
$$

for model 4. The temperature dependence of $Y_{n i}$ can now be easily factored out of the differentiation sign on the right-hand side of Eq. (36). In this way, one obtains the following asymptote for $\omega$,

$$
\omega \propto \frac{\sqrt{1-T^{\infty} / T_{c n}^{\infty}(0)}}{R_{0}} .
$$

Now, employing Eqs. (32) and (33), one finds for model 3

$$
\omega \propto\left(1-\frac{T^{\infty}}{T_{c n}^{\infty}(0)}\right)^{1 / 4} \rightarrow 0, \quad T^{\infty} \rightarrow T_{c n}^{\infty}(0),
$$

and for model 4

$$
\omega \rightarrow \text { finite, } \quad T^{\infty} \rightarrow T_{c n}^{\infty}(0)
$$

It should be emphasized that, owing to a weak dependence of the function $F_{n i}\left(r, T^{\infty}\right)$ on $T^{\infty}$, the eigenfrequencies for the model 4 do not tend to a constant, but remain weakly dependent on temperature. One can verify that the asymptotes obtained for all the models well describe our numerical results presented in Fig. 3.

In this study, we only consider profiles $T_{c n}^{\infty}(\rho)$ that have a maximum at densities higher than, or equal to the central stellar density $\rho_{c}$. This enables us to consider only two regions in the star: inner superfluid and outer normal. However, a situation in which the maximum lies at $\rho<\rho_{c}$ can easily be imagined. In this case, there will be generally three regions in a star: inner and outer normal regions and a superfluid region in between. The size $R_{0}$ of the superfluid region will decrease with increasing temperature in the same manner as in model $4, R_{0} \propto\left(1-T^{\infty} / T_{c n \text { max }}^{\infty}\right)^{1 / 2}($ where $T_{c n \text { max }}^{\infty}$ is the value of $T_{c n}^{\infty}$ at the maximum). Therefore, at $T^{\infty} \rightarrow T_{c n \max }^{\infty}$ the eigenfrequencies for such star will have the same asymptotes as for the model $4, \omega \rightarrow$ finite. The spectrum for a three-layer star is analyzed in Ref. [43].

\section{EIGENFUNCTIONS}

In this section we compare the main properties of superfluid and normal pulsation modes. For that we analyze the eigenfunctions for both types of modes. For brevity, we consider only the third model.

Figure 5 shows the eigenfunctions $\xi, z_{n}, z_{p}$, and $\xi_{b}$ (normalized to the stellar radius $R$ ), calculated at $T^{\infty}=$ $1.2 \times 10^{8} \mathrm{~K}$ versus $r$ (in units of $R$ ). The oscillation amplitude was chosen in such a way that the energy of radial pulsations is $E_{\text {puls }}=10^{48} \mathrm{erg}$ [see Eq. (44) for the definition of $E_{\text {puls }}$ ]. For a fundamental mode of a normal star this energy corresponds to an oscillation amplitude $\xi \sim 10^{-3} r$. It could be also interesting to note that this energy is of the order of the thermal energy of a normal star with $T^{\infty}=10^{9} \mathrm{~K}[44]$. Each mode (I,...,VI) in Fig. 5 is plotted in a separate panel. The boundary $R_{0}$ between the superfluid and normal regions, and the crust-core boundary $R_{\text {cc }}$, are indicated by vertical dotted lines. The solid line corresponds to the Lagrangian displacement $\xi$ of the nonsuperfluid component; the dashed and dot-dashed lines correspond to relative Lagrangian displacements $z_{n}$ and $z_{p}$, respectively; the heavy solid line - to the Lagrangian displacement $\xi_{b}$, which is proportional to the baryon current density. We do not plot $z_{n}$ in the nonsuperfluid region of the star since all neutrons are unpaired there. We also do not plot $z_{p}$ in the crust, because it contains no free protons. The quantity $\xi_{b}$ in the normal region of the star coincides with $\xi$.

At the chosen temperature $\left(T^{\infty}=1.2 \times 10^{8} \mathrm{~K}\right)$, the modes I, III, IV, and VI experience oscillations of the superfluid type and are approximately described by Eq. (27). The modes II and V are of normal type and are approximately described by Eq. (25); their eignefrequencies roughly coincide with the first and second modes of a normal star of the same mass. It can be seen that, as expected, the baryon current in superfluid oscillations, characterized by the quantity $\xi_{b}$, is almost zero.

It is noteworthy that the crust of a star, experiencing superfluid oscillations, remains nearly at rest, i.e., pulsations are mostly localized in the superfluid region. This point is illustrated in Fig. 6, which presents the ratio of average oscillation energy densities in the nonsuperfluid $\varepsilon_{\text {norm }}$ and superfluid $\varepsilon_{\text {sff }}$ regions as a function of $T^{\infty}$ for the first six pulsation modes $(\mathrm{I}, \ldots, \mathrm{VI})$. Modes I, II, and III are shown, respectively, by the solid, dotted, and dashed lines in the left panel; the other modes are shown in the right panel. The thin solid curve is plotted for the first two modes of a normal star $\left(\mathrm{I}_{\mathrm{nfh}}\right.$ and $\left.\mathrm{II}_{\mathrm{nfh}}\right)$. The latter modes depend on temperature only because of the variation of $R_{0}$ with $T^{\infty}$, which is artificially assumed to be the same as for a superfluid star.

It can be seen from Fig. 6 that, for normal-type oscillations, the ratio $\varepsilon_{\text {norm }} / \varepsilon_{\text {sfl }}$ coincides very well with the result for normal star. However, immediately after the star starts to experience pulsations of the superfluid type, $\varepsilon_{\text {norm }} / \varepsilon_{\text {sfl }}$ 

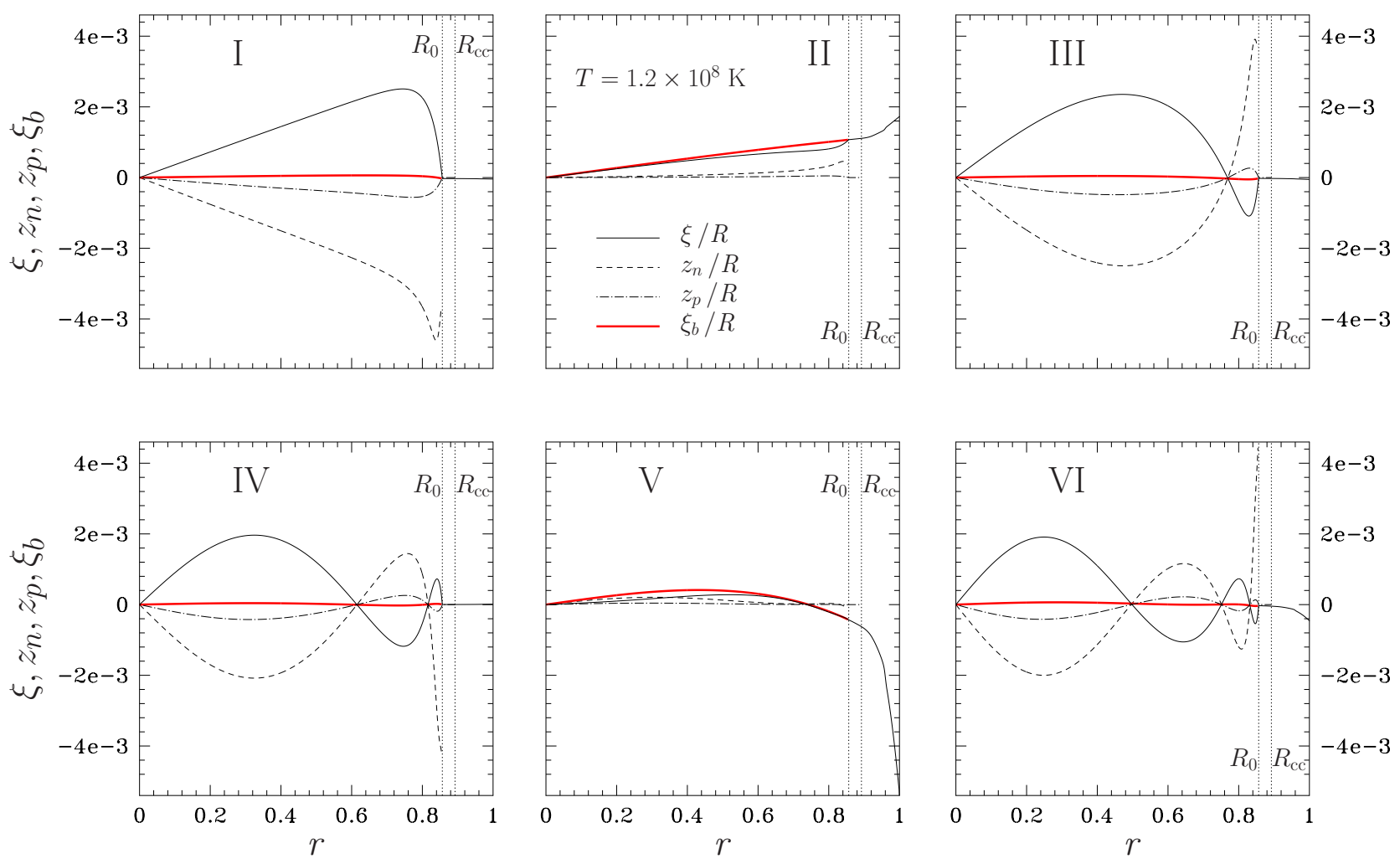

FIG. 5: Eigenfunctions $\xi, z_{n}, z_{p}$, and $\xi_{b}$ (normalized so that $E_{\text {puls }}=10^{48} \mathrm{erg}$ ) of a superfluid NS at $T^{\infty}=1.2 \cdot 10^{8} \mathrm{~K}$ versus $r$ (in units of $R$ ), calculated for the first six pulsation modes $(\mathrm{I}, \ldots, \mathrm{VI}$ ) of the model 3. Solid curves correspond to $\xi$, dashed to $z_{n}$, dot-dashed - to $z_{p}$, heavy solid lines show $\xi_{b}$. Vertical dots indicate the crust-core boundary and the superfluid-normal interface.

falls nearly to zero, so that almost all pulsation energy will be localized within the superfluid region. Thus, we see that the superfluid pulsation modes practically do not appear on the stellar surface and therefore it should be difficult to observe them by detecting the modulation of electromagnetic radiation from the star.

\section{DAMPING TIMES}

In this section we calculate characteristic damping times for radial pulsations due to the shear viscosity and nonequilibrium reactions of mutual particle transformations. It is well known that such reactions generate an effective bulk viscosity (see, e.g., [11, 45] and references therein).

The e-folding time is defined as

$$
\tau=-\frac{2 E_{\text {puls }}}{\left\langle\dot{E}_{\text {puls }}\right\rangle}
$$

where $\left\langle\dot{E}_{\text {puls }}\right\rangle$ is the damping rate for $E_{\text {puls }}$, averaged over the period $2 \pi / \omega$. Here and below, the angular brackets denote averaging over the pulsation period.

The expression for the pulsation energy of a superfluid symmetric nonrotating star has the form

$$
\begin{array}{r}
E_{\text {puls }}=\frac{1}{2} \int \omega^{2}\left[\mu_{i 0} \mu_{k 0} Y_{i k} z_{i(a)} z_{k(a)}+\mu_{i 0} \mu_{k 0} Y_{i k} \xi_{(a)}\left(z_{i(a)}+z_{k(a)}\right)+\left(P_{0}+\varepsilon_{0}\right) \xi_{(a)}^{2}\right] \\
\times 4 \pi r^{2} \mathrm{e}^{3 \lambda_{0} / 2-\nu_{0} / 2} d r,
\end{array}
$$

where $z_{i(a)}, z_{k(a)}$, and $\xi_{(a)}$ are the amplitudes of $z_{i}, z_{k}$, and $\xi$, respectively. Using the symmetry of the matrix $Y_{i k}$, 

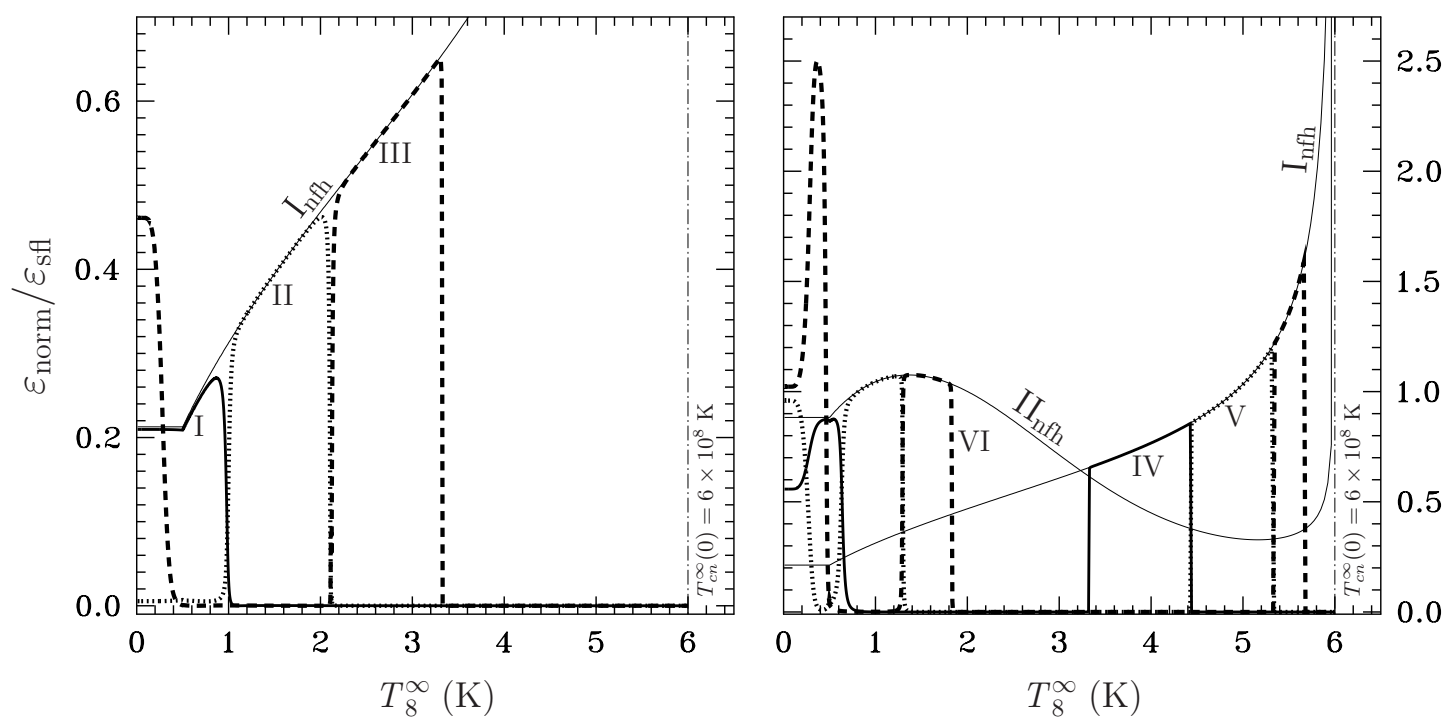

FIG. 6: The ratio of the pulsation energy densities in the nonsuperfluid and superfluid regions of a star versus $T_{8}^{\infty}$, calculated for model 3 (see the text). The left panel shows the first three modes (I, II, and III), the right panel shows the modes IV, V, and VI. Solid thin lines present the same ratio for the first two pulsation modes of a normal star $\left(\mathrm{I}_{\mathrm{nfh}}\right.$ and $\left.\mathrm{II}_{\mathrm{nfh}}\right)$. The redshifted neutron critical temperature $T_{c n}^{\infty}$ in the center of the star is indicated by the vertical dot-dashed lines.

the quasineutrality condition (12), and the definition (11) of $\xi_{b}$, we can simplify the expression for $E_{\text {puls }}$,

$$
\begin{array}{r}
E_{\text {puls }}=\frac{1}{2} \int \omega^{2}\left[\mu_{n 0} \mu_{k 0} Y_{n k} z_{k(a)}\left(z_{n(a)}-\frac{\mu_{i 0} Y_{n i} z_{i(a)}}{n_{b 0}}\right)+n_{b 0} \mu_{n 0} \xi_{b(a)}^{2}\right] \\
\times 4 \pi r^{2} \mathrm{e}^{3 \lambda_{0} / 2-\nu_{0} / 2} d r .
\end{array}
$$

For a stellar model considered here, the direct Urca process is forbidden, and the only effective processes of particle transformations are the neutron and proton branches of the modified Urca process,

$$
\begin{array}{ll}
n+n \rightarrow p+n+e^{-}+\bar{\nu}_{e}, & p+n+e^{-} \rightarrow n+n+\nu_{e} \\
n+p \rightarrow p+p+e^{-}+\bar{\nu}_{e}, & p+p+e^{-} \rightarrow n+p+\nu_{e} .
\end{array}
$$

These reactions lead to dissipation of $E_{\text {puls }}$ and to heating of the star. Assuming that pulsations are subthermal $(\delta \mu \ll$ $k T$ ), the conversion rate of the pulsation energy into the thermal energy (per unit volume) via the nonequilibrium reactions (46) and (47), is given by [17, 31, 44]

$$
Q_{\mathrm{bulk}}=\lambda \delta \mu^{2},
$$

where the chemical potential imbalance $\delta \mu$ can be expressed in terms of the above-calculated Lagrangian displacements $\xi, z_{n}$, and $z_{p}$ with the help of Eqs. (15), (17), and (18); and $\lambda \equiv \lambda_{1}+\lambda_{2}$ is the sum of the reaction rates (46) and (47). The reaction rates and the corresponding reduction factors were calculated for the modified Urca process in Ref. [46] (see also Ref. [47], in which a correction was introduced to the reaction rate for the proton branch).

The energy converted into heat per unit volume per second due to the shear viscosity is given by the same formula as for normal matter [4],

$$
Q_{\text {shear }}=\frac{\eta}{3} \omega^{2} \frac{\mathrm{e}^{-\nu_{0}}}{r^{2}}\left(-2 r \frac{\mathrm{d} \xi}{\mathrm{d} r}+2 \xi+r \xi \frac{\mathrm{d} \nu_{0}}{\mathrm{~d} r}\right)^{2}
$$

where $\eta$ is the shear viscosity coefficient. In our numerical calculations we used $\eta$ from [48]. Because the shear viscosity is mostly determined by the electron-electron and electron-proton collisions, we neglect the contribution of neutron collisions and use equation (37) from [48] when calculating $\eta$. In addition, we use equation (83) from the same reference to take into account the reduction of the particle collision frequency (and, consequently, the increase in the shear viscosity) by the proton superfluidity. 

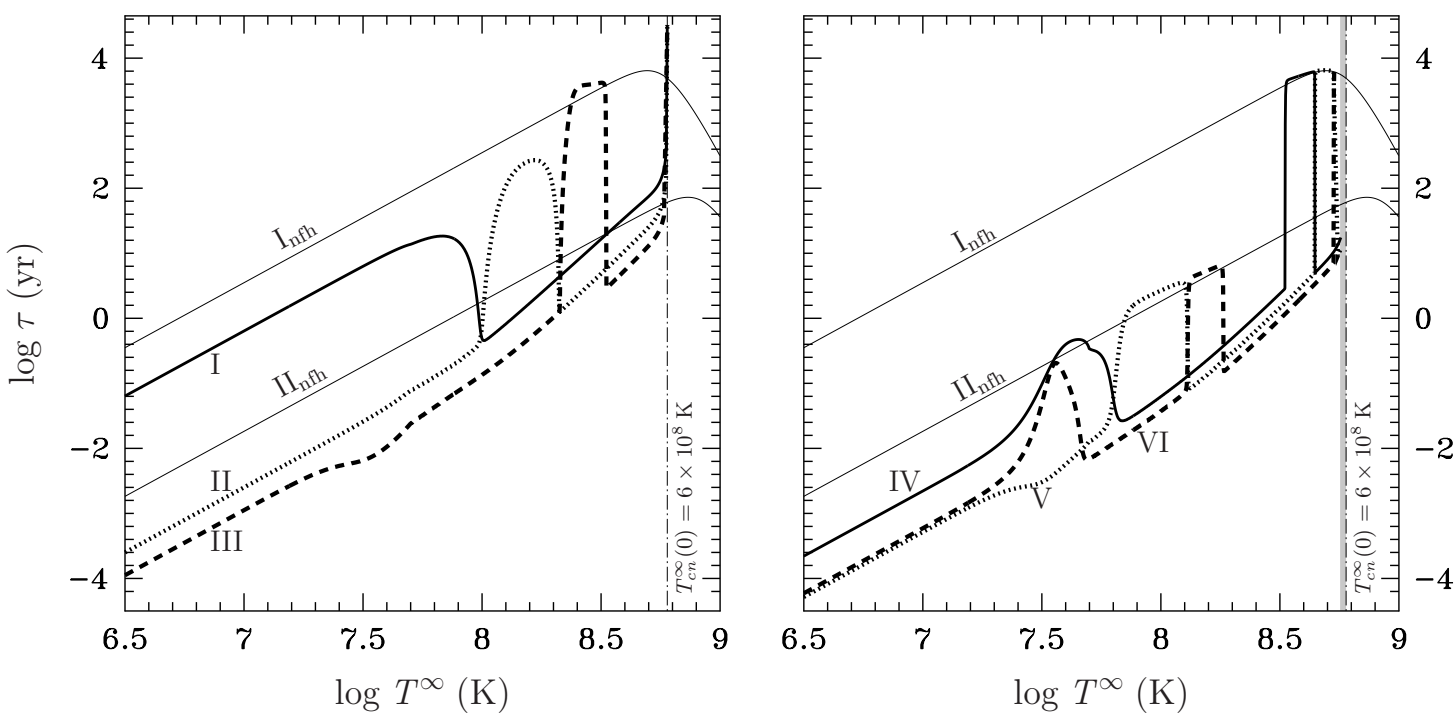

FIG. 7: Damping time $\tau$ versus $T^{\infty}$ for the first six pulsation modes of model 3 (see the text). The thin solid lines indicate $\tau$ for the first two modes of a normal star $\left(\mathrm{I}_{\mathrm{nfh}}\right.$ and $\left.\mathrm{II}_{\mathrm{nfh}}\right)$. Because of numerical problems $\tau$ was not calculated in the filled region. Other notations are the same as in Fig. 6.

To obtain the dissipation rate of the pulsation energy, one has to average Eqs. (48) and (49) over the pulsation period and then to integrate them over the stellar volume [44],

$$
\left\langle\dot{E}_{\text {puls }}\right\rangle=-\int\left(\left\langle Q_{\text {shear }}\right\rangle+\left\langle Q_{\text {bulk }}\right\rangle\right) \mathrm{e}^{\nu_{0}} 4 \pi r^{2} \mathrm{e}^{\lambda_{0} / 2} d r
$$

Having thus expressed all the quantities in Eq. (43) in terms of the already calculated eigenfunctions, we can present numerical results for the e-folding times. As in Sec. V, we consider only the model 3.

Figure 7 presents $\tau$ as a function of $T^{\infty}$, for the first six pulsation modes (three modes in each panel). As in Fig. 6 , the modes are shown by heavy solid, dotted, and dashed lines. Thin solid lines show $\tau$ calculated for the first two modes $\mathrm{I}_{\mathrm{nfh}}$ and $\mathrm{II}_{\mathrm{nfh}}$ by using normal-fluid (ordinary) hydrodynamics. In these calculations, superfluidity was taken into account only at calculating the kinetic coefficients (such an approximation is frequently employed in the literature to estimate the effect of superfluidity on the dissipative processes in NSs). The damping times were not calculated for the modes IV, V, and VI in the immediate vicinity of $T_{c n}^{\infty}(0)$ because of problems with numerical calculations (this region is filled grey).

It follows from Figs. 3 and 7 that $\tau$ for normal-type oscillations may differ severalfold from that calculated using the normal-fluid hydrodynamics. The difference is stronger at lower temperatures. This fact may seem surprising if we recall that the eigenfrequencies and eigenfunctions $\xi_{b}(r)$ approximately coincide for normal modes and for corresponding modes of a nonsuperfluid star. To explain the fact, one should notice that the function $\xi(r)$ generally differs in superfluid and normal stars. In additon, the relative motion of superfluid components with respect to the normal one $\left(z_{i} \neq 0\right)$ also modifies $\tau$.

Superfluid pulsation modes are damped faster than the normal modes, so that $\tau$ drops by $1-3$ orders of magnitude each time the star switches (e.g., in the course of thermal evolution) from normal- to superfluid-type regime of pulsations (see Figs. 3 and 7). To explain such a fast damping, let us note that it occurs (for $T^{\infty} \lesssim 6 \times 10^{8} \mathrm{~K}$ ) mainly due to the electron shear viscosity. For a nonsuperfluid matter the electron shear viscosity coefficient $\eta_{e}$ is smaller in the outer stellar layers than in the inner layers. Proton superfluidity further increases $\eta_{e}$ in the inner layers of the star. Now, if one looks at Fig. 5, one will notice that superfluid modes are localized in the superfluid core, while the amplitude of normal modes (modes II and V in that figure) grows away from the stellar center (with the maximum in the crust, where $\eta_{e}$ is small). In view of these facts the reason for the difference of damping times becomes clear; quantitatively, this result follows from a careful examination of Eqs. (43), (49), and (50).

In order to understand the limiting behavior of $\tau$ at high temperatures $\left[T^{\infty} \rightarrow T_{c n}^{\infty}(0)\right]$, let us analyze its asymptotes for different models. As already discussed in Sec. IV, any given mode near the neutron critical temperature experiences superfluid-type pulsations, approximately described by Eq. (27). For this type of pulsations $\xi_{b}$ is small, 
$\xi_{b} \ll \mu_{k 0} Y_{n k} z_{k} / n_{b}$, so that one derives from Eq. (45) the following estimate for $E_{\text {puls }}$ at $T^{\infty} \rightarrow T_{c n}^{\infty}(0)$

$$
E_{\mathrm{puls}} \sim \omega^{2} \mu_{n 0} \mu_{k 0} Y_{n k} \widetilde{z}_{k(a)}\left[\widetilde{z}_{n(a)}-\frac{\mu_{i 0} Y_{n i} \widetilde{z}_{i(a)}}{n_{b 0}}\right] R_{0}^{3} .
$$

Here and below, we neglect the factors of the order of unity in all formulas and replace integration over the stellar volume with the factor $R_{0}^{3}$; the quantities $\widetilde{z}_{n(a)}$ and $\widetilde{z}_{p(a)}$ in Eq. (51) denote $z_{n(a)}$ and $z_{p(a)}$, averaged over the superfluid region. Taking into account that for superfluid modes $\xi \approx-\mu_{k 0} Y_{n k} z_{k} / n_{b 0}$ one obtains, using Eqs. (48) and (49), the following estimate for the dissipation rate due to, respectively, the shear viscosity and nonequilibrium modified Urca reactions (bulk viscosity)

$$
\begin{array}{r}
\left\langle\dot{E}_{\text {puls sh }}\right\rangle \sim-\eta \omega^{2}\left(\frac{\mu_{k 0} Y_{n k} \widetilde{z}_{k(a)}}{n_{b 0}}\right)^{2} R_{0}, \\
\left\langle\dot{E}_{\text {puls b }}\right\rangle \sim-\lambda n_{e 0}^{2}\left(\frac{\mu_{k 0} Y_{n k} \widetilde{z}_{k(a)}}{n_{b 0}}\right)^{2}\left(\frac{\partial \delta \mu}{\partial n_{e}}\right)^{2} R_{0} .
\end{array}
$$

In view of Eqs. (51)-(531) one finds for the corresponding e-folding times,

$$
\begin{gathered}
\tau_{\text {sh }} \equiv-\frac{2 E_{\text {puls }}}{\left\langle\dot{E}_{\text {puls sh }}\right\rangle} \sim \frac{\mu_{n 0}\left[\widetilde{z}_{n(a)}-\mu_{i 0} Y_{n i} \widetilde{z}_{i(a)} / n_{b 0}\right] R_{0}^{2} n_{b 0}^{2}}{\eta \mu_{k 0} Y_{n k} \widetilde{z}_{k(a)}}, \\
\tau_{\mathrm{b}} \equiv-\frac{2 E_{\text {puls }}}{\left\langle\dot{E}_{\text {puls b }}\right\rangle} \sim \frac{\omega^{2} \mu_{n 0}\left[\widetilde{z}_{n(a)}-\mu_{i 0} Y_{n i} \widetilde{z}_{i(a)} / n_{b 0}\right] R_{0}^{2} n_{b 0}^{2}}{\lambda n_{e 0}^{2} \mu_{k 0} Y_{n k} \widetilde{z}_{k(a)}\left(\partial \delta \mu / \partial n_{e}\right)^{2}} .
\end{gathered}
$$

Now, using the asymptote (29) for $Y_{i k}$ and the asymptotes for $\omega$ and $R_{0}$, derived in Sec. IV, one gets for $\tau_{\text {sh }}$ and $\tau_{\mathrm{b}}$ in the limit $T^{\infty} \rightarrow T_{c n}^{\infty}(0)$,

$$
\begin{aligned}
\tau_{\text {sh }} & \propto \frac{R_{0}^{2}}{Y_{n n}} \rightarrow\left(1-\frac{T^{\infty}}{T_{c n}^{\infty}}\right)^{\alpha}, \\
\tau_{\mathrm{b}} & \propto \frac{\omega^{2} R_{0}^{2}}{Y_{n n}} \rightarrow \text { finite }
\end{aligned}
$$

Here $\alpha=-1$ for the first model, $\alpha=1 / 2$ for the second, $\alpha=-1 / 2$ for the third, and $\alpha=0$ for the fourth model. The damping time $\tau_{\mathrm{b}}$ tends to some finite value for all the models. For the third model, presented in Fig. 7, the damping rate due to the shear viscosity drops as $\sqrt{1-T^{\infty} / T_{c n}^{\infty}}$ at $T^{\infty} \rightarrow T_{c n}^{\infty}(0)$ (that is, $\tau_{\text {sh }}$ increases). Hence, very close to $T_{c n}^{\infty}(0), 1 / \tau_{\mathrm{sh}} \ll 1 / \tau_{\mathrm{b}}$ so that $\tau=\tau_{\mathrm{sh}} \tau_{\mathrm{b}} /\left(\tau_{\mathrm{sh}}+\tau_{\mathrm{b}}\right)$ is bounded from above by $\tau_{\mathrm{b}}$ (this region of $T^{\infty}$ is too narrow to be visible in the figure).

\section{SUMMARY}

This work analyses the effects of finite temperatures on the oscillations of superfluid NSs. The most simple case of a radially pulsating nonrotating star is considered in the frame of general relativity. The impact of two temperature effects on the pulsations is examined. The first effect concerns the temperature dependence of the relativistic entrainment matrix $Y_{i k}$ - one of the important ingredients of superfluid hydrodynamics. The second effect consists in decreasing of the size of superfluid region with increasing temperature. The first effect was studied in GA06, the second - in Refs. 23, 28, 29].

We considered four different models which allow for one or both of these effects, and employ various profiles $T_{c n}^{\infty}(r)$ of neutron critical temperature. We calculated pulsation spectra for these models. For each model the presence of two distinct classes of oscillation modes, the so called normal and superfluid modes, is revealed. The frequencies of normal modes almost coincide with the corresponding frequencies of a nonsuperfluid star. On the contrary, the frequencies of superfluid modes strongly depend on temperature and show different type of behavior depending on a model. It is demonstrated that the model which takes into account only the second temperature effect (dependence of the size of superfluid region on $T$ ) but ignores the first effect, yields qualitatively incorrect spectra.

Using the approach developed in Refs. [33, 34], we found the asymptotes for eigenfrequencies at $T^{\infty} \rightarrow T_{c n}^{\infty}(0)$, which are in a good agreement with the results of numerical calculations.

In addition, we analyzed the eigenfunctions for the first six oscillation modes. It is shown that the baryon current density is almost unperturbed in superfluid modes. Moreover, these modes are mainly localized in the core and 
practically do not appear on the neutron-star surface. As a consequence, it might be difficult to detect them by observing modulation of the electromagnetic radiation from the star.

We also calculated the characteristic damping times $\tau$ for radial pulsations due to the shear viscosity and nonequilibrium modified Urca processes and analyzed the asymptotes of $\tau$ at $T^{\infty} \rightarrow T_{c n}^{\infty}(0)$. It is shown that damping times, calculated using the ordinary (nonsuperfluid) hydrodynamics may differ severalfold from $\tau$ calculated for normal modes (even though the eigenfrequencies of normal modes almost coincide with the corresponding eigenfrequencies of a nonsuperfluid star). It is also demonstrated that, generally, damping of superfluid-type pulsations occurs by 1-3 orders of magnitude faster than damping of normal-type pulsations.

\section{Acknowledgments}

We thank A.I. Chugunov for valuable comments. This study was supported by the Dynasty foundation, Ministry of Education and Science of Russian Federation (contract No. 11.G34.31.0001 with SPbSPU and leading scientist G.G. Pavlov), RFBR 11-02-00253-a, and FASI (grant NSh-3769.2010.2).

[1] T. E. Strohmayer and A. L. Watts, Astrophys. J. 632, 111 (2005).

[2] G. L. Israel, T. Belloni, L. Stella, Y. Rephaeli, D. E. Gruber, P. Casella, S. Dall'Osso, N. Rea, M. Persic, and R. E. Rothschild, Astrophys. J. 628, L53 (2005).

[3] N. Andersson and K. D. Kokkotas, Int. J. Mod. Phys. D10, 381 (2001).

[4] N. Andersson, Class. Quantum Grav. 20, R105 (2003).

[5] B. Owen, Phys. Rev. D82, 104002 (2010).

[6] B. Abbott, R. Abbott, R. Adhikari, J. Agresti, P. Ajith, B. Allen, R. Amin, S. B. Anderson, W. G. Anderson, M. Arain, and 437 coauthors, Phys. Rev. D76, 062003 (2007).

[7] D. G. Yakovlev, K. P. Levenfish, and Yu. A. Shibanov, Phys. Usp. 42, 737 (1999).

[8] U. Lombardo and H.-J. Schulze, Lect. Notes Phys. 578, 30 (2001).

[9] D. G. Yakovlev and C. J. Pethick, Annu. Rev. Astron. Astrophys. 42, 169 (2004).

[10] N. Andersson and G. L. Comer, Mon. Not. R. Astron. Soc. 328, 1129 (2001).

[11] M.E. Gusakov, Phys. Rev. D76, 083001 (2007).

[12] M. E. Gusakov and N. Andersson, Mon. Not. R. Astron. Soc. 372, 1776 (2006).

[13] G. L. Comer, D. Langlois, and L. M. Lin, Phys. Rev. D60, 104025 (1999).

[14] L. Lindblom and G. Mendell, Astrophys. J. 421, 689 (1994).

[15] I. M. Khalatnikov, An Introduction to the Theory of Superfluidity (Addison-Wesley, New York, 1989).

[16] S. J. Putterman, Superfluid Hydrodynamics (North-Holland, Amsterdam, 1974).

[17] L. D. Landau and E. M. Lifshitz, Fluid mechanics, Course of theoretical physics, (Pergamon Press, Oxford, 1987).

[18] R. I. Epstein, Astrophys. J. 333, 880 (1988).

[19] L. Lindblom and G. Mendell, Phys. Rev. D61, 104003 (2000).

[20] N. Andersson, G. L. Comer, and D. Langlois, Phys. Rev. D66, 104002 (2002).

[21] N. Andersson and G. L. Comer, Class. Quant. Grav., 18, 969 (2001).

[22] S. Yoshida and U. Lee, Phys. Rev. D67, 124019 (2003).

[23] L.-M. Lin, N. Andersson, and G. L. Comer, Phys. Rev. D78, 083008 (2008).

[24] R. Prix, G. L. Comer, and N. Andersson, Mon. Not. R. Astron. Soc. 348, 625 (2004).

[25] S. Yoshida and U. Lee, Mon. Not. R. Astron. Soc. 344, 207 (2003).

[26] T. Sidery, N. Andersson, and G. L. Comer, Mon. Not. R. Astron. Soc. 385, 335 (2008).

[27] U. Lee, Astron. Astrophys. 303, 515 (1995).

[28] B. Haskell, N. Andersson, and A. Passamonti, Mon. Not. R. Astron. Soc. 3971464 (2009).

[29] B. Haskell and N. Andersson, Mon. Not. R. Astron. Soc. 4081897 (2010).

[30] M. E. Gusakov and E. M. Kantor, Phys. Rev. D78, 083006 (2008).

[31] E. M. Kantor and M. E. Gusakov, Phys. Rev. D79, 043004 (2009).

[32] M. E. Gusakov, E. M. Kantor, and P. Haensel, Phys. Rev. C80, 015803 (2009).

[33] M. E. Gusakov and E. M. Kantor, Phys. Rev. D83, 081304 (R) (2011).

[34] M. E. Gusakov and E. M. Kantor, in preparation (2011).

[35] We warn the reader not to confuse this definition of $n_{l}$ with the other definition, $n_{l}=-\sqrt{j_{\mu(l)} j_{(l)}^{\mu}}$, which is frequently used in the literature devoted to pulsations of superfluid NSs (see, e.g., [22, 23, 28, 29]). Generally, these two definitions are not equivalent. However, they are equivalent if one studies pulsations in the linear approximation and, in addition, assumes that an unperturbed star was initially at rest [i.e., $u^{\mu}=(1,0,0,0)$ and $w_{(i)}^{\mu}=0$ ].

[36] A. F. Andreev and E. P. Bashkin, Zh. Eksp. Teor. Fiz., 69, 319 (1975).

[37] H. Heiselberg and M. Hjorth-Jensen, Astrophys. J. 525, L45 (1999). 
[38] Akmal A., Pandharipande V.R., Ravenhall D. G., Phys. Rev. C58, 1804 (1998).

[39] S. Chandrasekhar, Astrophys. J. 140, 417 (1964).

[40] J.W. Negele, D. Vautherin, Nucl. Phys. A207, 298 (1973).

[41] R. Prix and M. Rieutord, Astron. Astrophys. 393, 949 (2002).

[42] M. E. Gusakov and P. Haensel, Nucl. Phys. A761, 333 (2005).

[43] A. I. Chugunov and M. E. Gusakov, to be submitted to MNRAS (2011).

[44] M. E. Gusakov, D. G. Yakovlev, and O. Y. Gnedin, Mon. Not. R. Astron. Soc. 361, 1415 (2005).

[45] H. Dong, N. Su, and Q. Wang, Journal of Physics G: Nuclear and Particle Physics, Volume 34, pp. S643-S646 (2007).

[46] P. Haensel, K. P. Levenfish, and D. G. Yakovlev, Astron. Astrophys. 372, 130 (2001).

[47] P. Haensel, K. P. Levenfish, and D. G. Yakovlev, Astron. Astrophys. 381, 1080 (2002).

[48] P. S. Shternin and D. G. Yakovlev, Phys. Rev. D78, 063006 (2008). 